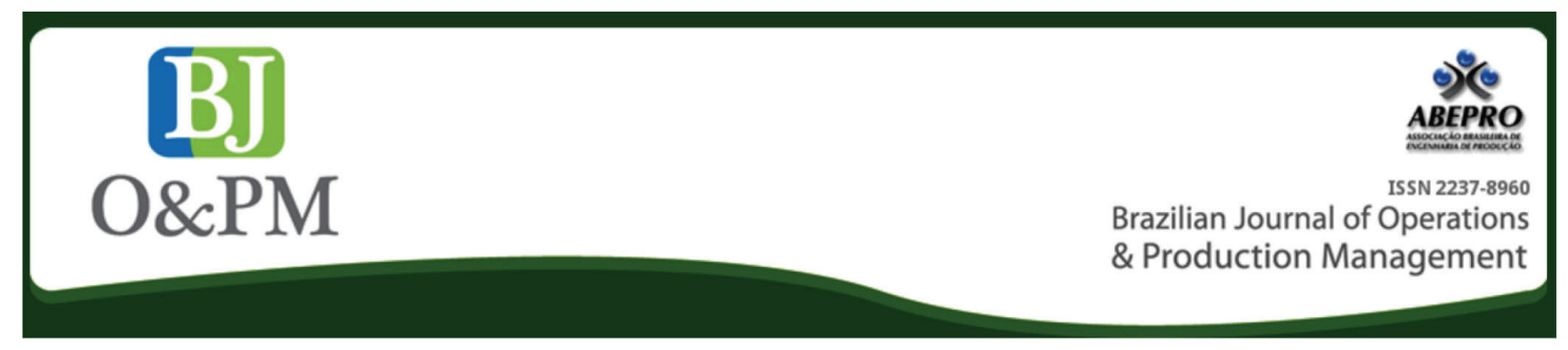

\title{
THE BLOCKCHAIN TECHNOLOGY ON THE MUSIC INDUSTRY
}

Luis Claudio Arcos

Icarcos@gmail.com

Poliphonia, Rio de Janeiro, Rio de Janeiro, Brazil.

\begin{abstract}
Blockchain does appear to have a transformative potential for creativity industries. Mougayar (2016) states that blockchain technology is as important as the World Wide Web. Although this technology is in its early days, it holds the potential to drastically change the supply chain. Organizations such as Open Music Initiative have already started to explore these possibilities. This article aims to analyze how might blockchain technology emerges as a disruptive force on the music industry.
\end{abstract}

Keywords: Blockchain; Music; Interoperability; Decentralized; Creative Industries; Copyright. 


\section{INTRODUCTION}

The Internet has rearranged the music industry. The reorganization of the value chain has speeded up since the emergence of streaming services. Music consumption has shifted from "ownership" to "access" and as the roles of creators and consumers have blurred, the industry's traditional revenue base and money flows have been disrupted.

Because music consumption and creation is changing rapidly, there is a need to track song contribution and attribution beyond just streaming plays and downloads for new creative expressions. At its simplest, this will be consumer-generated content such as mash ups - mixing songs together or adding songs to other digital content as film clips or videos.

"New technologies can be applied to radically simplify the way music rights owners are identified and compensated, resulting in sustainable business models for artists, entrepreneurs, and music businesses alike" (Panay, 2016).

Although the blockchain technology is in its early days, it holds the potential to change the entire supply chain. Organizations such as Berklee's Open Music Initiative (OMI) have managed to bring together almost every party in the industry to explain the reason for exploring and getting involved with the technology.

The article aims to analyze how the blockchain technology might emerge as a disruptive force in the music industry, offering a view as an OMI's partner. It analyzes what technological changes mean for the increasing and distributing music industry revenue. How artists' revenue share may be affected. This article is divided into two core sections followed by a conclusion. The first section briefly explains blockchain technology. The second section focuses on how blockchain might impact the music industry. The final section provides some conclusions.

\section{WHAT IS BLOCKCHAIN?}

Blockchain is a distributed ledger. The technology emerged as the basis for cryptocurrency, Bitcoin. The technology is an ingenious invention of a person or a group of people known by the pseudonym, Satoshi Nakamoto. Many people believe that Nakamoto's most important innovation was not bitcoin itself, but its underlying blockchain technology.

The blockchain technology has some features that make it highly attractive as data storage:

- Data written to it is immutable and therefore provides as auditable record of events that cannot be modified.
- An exact copy is maintained in a large number of independent locations.

- There is no central point of failure.

By allowing digital information to be distributed but not copied, blockchain technology has created the backbone of a new type of internet. Mougayar (2016) claims that blockchain technology is as important as the World Wide Web, and arguably might give us back the Internet, in the way it was supposed to be: more decentralized, more open, more secure, more private, more equitable, and more accessible (Figure 1).

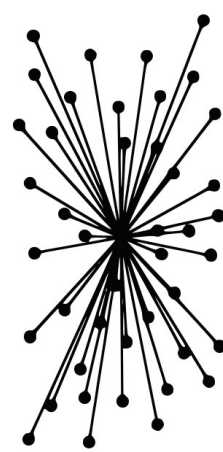

Centralized

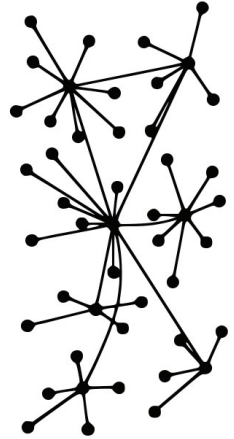

Decentralized

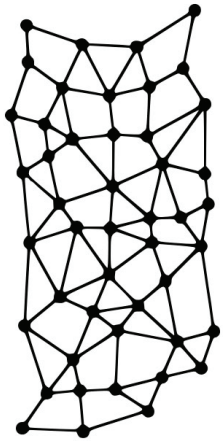

Distributed
Figure 1. Centralized, decentralized and distributed systems

Source: NetworkCultures.org

Rather than having a central administrator such as a traditional database, a distributed ledger has a network of chronologically involving replicated databases, synchronized by the use of the internet, and visible to anyone within the network.

The distributed, cryptographic and open nature of blockchain allows people to trust each other and transact peer-topeer, making the need for intermediaries obsolete.

Summing it up, it is a shared, auditable, complete history of all transactions. It is secured by cryptography and distributed verifiers of the history of all transaction.

Blockchain, then, refers to a technology rather than a particular product, and its applications are already diverse.

\section{HOW MIGHT IT TRANSFORM THE MUSIC INDUSTRY}

The music industry involves many entities. The supply chain involves Artists, Publishers, Labels or Record Com- 
panies, Retailers, Performance Rights Organization (PROs), Streaming Digital Service Providers. The internet has rearranged the music industry. The reorganization of the value chain has speeded up since the emergence of streaming services. Major labels have watched record sales dropping. Meanwhile, streaming services are growing in popularity (Figure 2) but drowning in lawsuits.

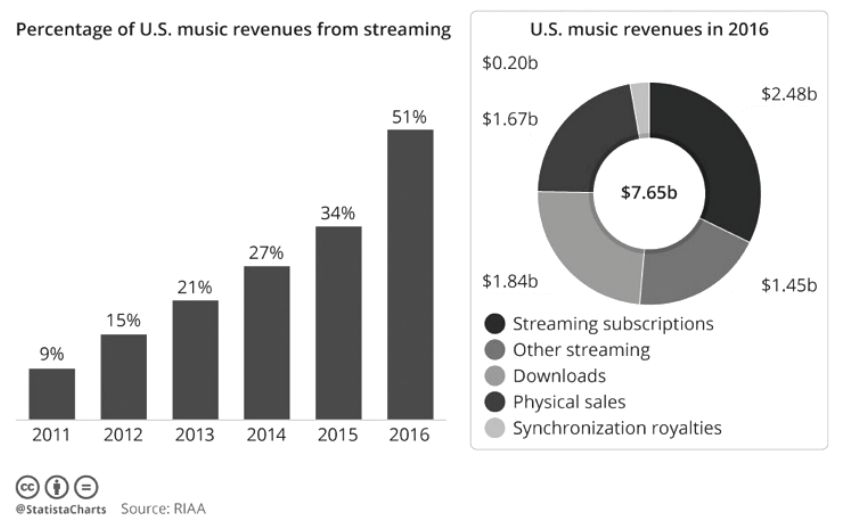

Figure 2. U.S. music revenues from streaming, 2011-2016 Source: RIAA (2017)

Nowadays, music can be streamed and downloaded at the click of a button, but payments to the people who make that music can be slow and not transparent.

A major pain point for creatives in the music industry such as songwriters, producers and musicians - is that they are the first to put in any of the work, and the last to ever see any profit. They have little or no information about how their royalty payments are calculated, and do not get access to valuable aggregate data about how and where people are listening to their music (Heap, 2017).

Blockchain technology offers transparency through the value chain. Musicians and managers can see exactly how much money they are owed. The technology could solve the issue of identifying a real copyright holder and ease the challenge of tracking derivative works through the value chain. This could make it possible to see not only the creators, but also those who were materially involved through a distributed ledger of metadata. This would create a peer-to-peer ecosystem for artists that enable them to control their own fate and receive the fair compensation for the value they create.

Currently, the main stakeholders in the industry are developing the open-source technology that will support blockchain development in the music industry. The Open Music Initiative (OMI) is led by the Berklee College of Music Institute for Creative Entrepreneurship. The initiative is a collaboration with the MIT Media Lab with support from several major music labels, streaming services, publishers, collection societies, and nearly other 60 founding entities. The OMI's mission is to promote and advance creation of open-source standards and innovation related to music to help assure proper compensation for all creators, performers, and right holders. OMI is not a database, but instead an open-source protocol to uniform identification of music right holders and creators.

For the first time since the advent of the digital era, there is consensus from nearly every organization and entity involved in the music industry - record labels, publishers, performing rights societies, streaming services, managers, artists, and startups - that addressing this on matter is ground zero. This consensus, coupled with the emergence of new technologies such as distributed ledgers that introduce new opportunities and possibilities; and, amplified by the collective will to make change and make this moment a unique for the music business - a once-a-generation opportunity to proactively design the future and not dwell on the failings of the past (Panay, 2016).

Berklee College of Music executed a Lab to pilot platforms based on blockchain technology. The OMI Lab hosted musicians, visual artists, software developers, and entrepreneurs from all over the world. The group has been working together, developing new startups prototypes for the music industry, exploring blockchain registration, and making suggestions for the development of the OMI API.

The globally renowned innovation and design firm, IDEO, has facilitated the summer lab. The group was assigned to tackle various problems and tasks within the music industry through IDEO's Human-Centered Design process.

These tasks included:

1. Cataloging, attributing, and distributing live DJ mixes.

2. Compensating musicians for visual works using their songs and data.

3. Identifying individuals for their contribution to single tracks in new works.

4. Commercializing mixtapes built from original material and back catalogs.

At the end of the Lab, the teams presented the following solutions: 
- DEEPDIVE: An organized platform where fans can view an artist's entire identity and share meaning behind the music.

- LÜM: A new service that allows for unique emotional moments in a live performance to be captured and analyzed for artists and businesses involved in the music industry.

- ECHOWE: A sample marketplace where copyright holders can set the intention of their music, so it can act on its own as if it were alive.

- $\quad$ FIBER: A media player that tells the holistic story of a song by bringing it to life in virtual reality.

Though the startups are based on embryonic business models it is possible to see the potential of blockchain technology in these areas:

- A networked database for music copyright information

- $\quad$ Fast and frictionless royalty payments

- Transparency through the value chain

- Alternative sources of capital

Any sample of recorded music has two copyrights: one for the phonogram, the sound recording itself, relating to performers and the record labels; another copyright for the work itself, words and music, relating to songwriters or composers and music publishers.

One of the biggest problems in the industry right now is that there is no global registry of music creatives and their works. Blockchain has the potential to provide a quicker and seamless experience for anyone involved with creating or interacting with music. Blockchain technology is potentially transformative in terms of functioning as both database and network (De Leon et Gupta, 2017).

In the digital era music is data. Metadata is data of data, that is, information about music. Metadata embedded into every piece of recorded music could include terms of use and contact details for the copyright holders, making it fairly easier to locate the owners of a piece of recorded music and to obtain license to use it. The idea is to give music a purpose, so it can act on its own as if it were alive. The gradual placing of copyright data on the blockchain could ultimately lead to the creation of one comprehensive copyright database for music (LO'Dair, 2016).

Smart contracts - contracts written on computer code - implemented via software, could allow music royalties to be administered almost instantaneously. Rather than passing through intermediaries, revenue from a stream or download could be distributed automatically between rights holders, according to agreements, as soon as the track is downloaded or streamed. "It's a simple question of attribution, and payments follow attribution" (Panay, 2016).

The low transaction costs of cryptocurrencies make micropayments feasible. In the streaming era, this is specifically useful given the small size of typical payments. Content creators would receive tips, even in very small sums.

Another interesting point is bringing transparency throughout the value chain once blockchain technology could radically transform a culture of non-disclosure agreements and 'black boxes'.

The ingenuity of Bitcoin lies in 'allowing any two willing parties to transact directly with each other without the need for a trusted third party' (Nakamoto, 2008, p. 1). Some people have suggested that blockchain technology could remove such third parties from the record industry. In practice, it is probable that intermediaries that add value will remain, though agreements may alter in the favor of artists and songwriters. Music publishers, for instance, might concentrate on data verification and the resolution of disputes; even if their administrative function is replaced by blockchain technology, their role in negotiating payments is likely to continue. Perhaps most significantly, royalty payments could be split almost instantly according to agreed percentages and be paid directly to artists and songwriters. Although third parties could still take a cut, they might occupy a different position in the value chain.

It is envisioned the possibility of a networked database of music copyright information, instant micropayments, transparency through the value chain and access to alternative sources of capital as the key potential benefits of blockchain technology for the music industry.

\section{CONCLUSION}

There is, currently, a huge buzz around blockchain technology. Much is speculated about its applications and, although the future of music is still not entirely clear, the potential of technology to dramatically alter the supply chain is undeniable.

Predictions of an immediate adoption by the industry are very optimistic. Some predict that mainstream adoption will take at least 10 years. Maybe in 5 years a "parallel alternative industry" of artists and independent label using blockchain technology will begin to affect the majors. Further research 
is needed on how blockchain technology can coexist with current contractual and copyright systems.

The impact of the blockchain technology on the entertainment and creative industries is just beginning. In the coming months new cases will continue to appear. It will take some key cases to prove to the industry that the technology is feasible. This paradigm shift will differentiate organizations in the market that are the first to innovate and create new and satisfying solutions. What could be some of these innovations? Media companies that use blockchain to generate efficiency can improve the attribution of rights offering an innovative way to differentiate attracting participants to the ecosystem.

There are, of course, challenges for widespread adoption. It may depend on blockchain technology to become "invisible", affecting the back end of the music transaction and not the user experience. Adoption by large corporations will depend on a clear value proposition: as a means of dealing with the volume of on-demand transmission data, for example, or a way to monetize derivative works and user-generated content. To reach critical mass it may also require not only support from distributors but also the involvement of top-level artists.

Recently Spotify has acquired the blockchain startup Mediachain Labs, whose team works on the development of technologies to connect artists and other rights holders with tracks hosted on the Spotify service. Medihain develops technologies that can assist, including a decentralized peer-to-peer database to connect applications with media and information about it, as well as an attribution engine for creators and a cryptocurrency that rewards creators for their works. The startup uses blockchain technology to solve attribution problems.

Understanding technology is essential for developing strategic planning that can help media companies stand out from the competition and potentially create a new level of collaboration and trust for the music industry of the future.

What is clear is that the potential of blockchain technology has just begun to be explored.

\section{REFERENCES}

De Leon, I.; Gupta, R (2017), The Impact of Digital Innovation and Blockchain on the Music Industry, Institutions for Development Sector, Competitiveness, Technology, and Innovation Division, Discussion Paper no IDB-DP-549, https:// publications.iadb.org/bitstream/handle/11319/8627/The-Impact-of-Digital-Innovation-and-Blockchain-on-the-Music-Industry.PDF (Access $2018 \mathrm{Jul}$ 17).

Heap, I. (2017), "Blockchain Could Help Musicians Make Money Again". Harvard Business Review, available at: https:// hbr.org/2017/06/blockchain-could-help-musicians-make-money-again (Access $2018 \mathrm{Jul}$ 17).

LO'Dair, M. (2016), Music on The Blockchain, Blockchain For Creative Industries Research Cluster Middlesex University, Report $n$ o 1, available at: https://www.mdx.ac.uk/_data/ assets/pdf_file/0026/230696/Music-On-The-Blockchain.pdf (Access $2018 \mathrm{Jul} 17$ ).

Mougayar, W. (2016), "The Business Blockchain: Promise, Practice, and Application of the Next Internet Technology", Wiley.

Nakamoto, S. (2008), Bitcoin: A Peer-to-Peer Electronic Cash System, available at: https://bitcoin.org/bitcoin.pdf (Access $2018 \mathrm{Jul}$ 17).

Panay, P. (2016), Why Us, Why Now: Convening the Open Music Initiative, BerkleelCE, available at: https://www.berklee. edu/panos-panay-open-music-initiative (Access 2018 Jul 17).

Recording Industry Association of America - RIAA (2017), U.S. Sales Database, available from: https://www.riaa.com/u-s-sales-database/ (Access $2018 \mathrm{Jul}$ 17).

Received: 02 Feb 2018

Approved: 25 Jul 2018

DOI: 10.14488/BJOPM.2018.v15.n3.a11

How to cite: Arcos, L. C. (2018), "The blockchain technology on the music industry", Brazilian Journal of Operations \& Production Management, Vol. 15, No. 3, pp. 439-443, available from: https://bjopm.emnuvens.com.br/bjopm/ article/view/449 (access year month day). 